\title{
Design guideline for designing single handle faucets based on the correlations between human lifestyles and design elements
}

\author{
Riku Takagi *, Ippei Shimizu ** and Noriko Hashida *** \\ * Graduate School of Engineering and Science, Shibaura Institute of Technology, 3-7-5, Toyosu, Koto-ku, Tokyo, Japan \\ md18050@shibaura-it.ac.jp \\ ** TAKAGI Co. Ltd., 2-4-51, Ishidaminami, Kitakyushu-shi, Fukuoka, Japan \\ simizu862@takagi.co.jp \\ *** Shibaura Institute of Technology, 3-7-5, Toyosu, Koto-ku, Tokyo, Japan \\ hashida@shibaura-it.ac.jp
}

\begin{abstract}
The reason why there are many kinds of single handle faucet designs, is because there are no design guidelines for their exterior design, correlated to the present diverged consumer values. This study is on making a design guideline for single handle faucets, based on the correlations between human lifestyles and design elements. First, we developed 7 basic formed single handle faucets and had a questionnaire survey on the test subject's basic attributes, lifestyle and image evaluation on the developed faucets. We used the mathematical quantification theory class III, to find out what kind of basic attributes and lifestyle segments, values most on the exterior designs when purchasing, and what kind of basic design configuration they prefer most. Secondly, in the same questionnaire survey, we made the test subjects evaluate 7 basic single handle faucet form's images and their purchase intentions, in 6 levels. We used the multiple regression analysis, to abstract the image words, which has a strong correlation with the purchase intention, regarding to the abstracted basic attributes and the lifestyle. Finally, we made a questionnaire survey on the images of the existing gooseneck-type single handle faucet with various design elements, and made the test subjects evaluate the images in 6 levels. We used the mathematical quantification theory class I, to abstract the design elements, which has a strong correlation with the test subject's purchase intention. Consequently, we were able develop a design guideline for designing single handle faucets, based on the correlations between human lifestyles and design elements.
\end{abstract}

Keywords: Single handle faucet, Human lifestyles, Diverse values, Design elements, Correlation

\section{Introduction}

This study is on finding the guidelines in designing single handle faucets, based on the correlation between human lifestyles and design elements. Various types of single handle faucet designs are being developed throughout the years. This is a serious matter to designers and to consumers, because it is hard in both developing and purchasing the best designs for fulfilling the diverged consumer values, nowadays. In the research of Forward CO. LTD., they have found out that the Japanese can be divided into 8 type of groups, based on their "values and consumption needs". These groups are called lifestyle segments [1]. Furthermore, in these lifestyle segments, there are several segments which makes the next trend, called the FLOW segment. Therefor, we examined that it is important to target the consumers who are grouped in the FLOW segment, when developing a new single handle faucet design. From our previous study, we found out that there are different emotional effects, regarding to each life style segment [2]. In this study, we used several questionnaire surveys, to find out the specific FLOW segment which most values in the exterior design of single handle faucets, and abstracted the design elements which has strong correlations with their purchase intentions, to make a design guideline.
2. Research on finding the lifestyle segment which values most on the exterior designs when purchasing

\subsection{Survey content and purpose}

A questionnaire survey was developed, in the purpose of finding the lifestyle segment which values most on the exterior designs when purchasing. We made the test subjects answer their basic attributes, awareness to housework, their values when purchasing products, the single handle faucet's design configuration with the highest purchase intention and 30 fixed questions to discriminate their lifestyle segments.

\subsection{Survey method}

We gathered 500 test subjects, and made them answer to the questionnaire survey above. First, we used the 30 fixed questions to discriminate the test subjects into 8 lifestyle segments. After that, we used the mathematical quantification theory class III, to analyze and abstract the basic attributes and lifestyle, values most on the designs of the single handle faucet when purchasing, and the basic design configuration they prefer most. 


\subsection{Result}

We were able to make a scatter plot from the result of the mathematical quantification theory class III, and succeeded to find out the inherent characteristics of the test subjects. Figure 1 shows the enlarged scatter plot focused on the lifestyle segment called "Pride". As the scatter plot shows, "Pride" is one of the FLOW segment, and has high awareness to housework such as cooking, dish washing and keeping their house clean. Moreover, we found out that this lifestyle segment values most in the exterior design of single handle faucets. The characteristic of "Pride" is shortly described as "wanting to show their life to other people". Therefor, it is speculated that the people with this characteristic have high awareness in keeping their house clean, prepared to be seen by other people at anytime. Furthermore, from this research, the design configuration that the "Pride with high awareness on housework" prefer most, is the type which is called the gooseneck faucet. The reason is speculated as, the needs in wanting to appeal their sense in design and the curved shape for easy cleanability. From these results, it is optimum to target "Pride with high awareness on housework", in case of designing a new single handle faucet emphasizing on the exterior design. Figure 2 shows the summary on the abstracted target attribute.

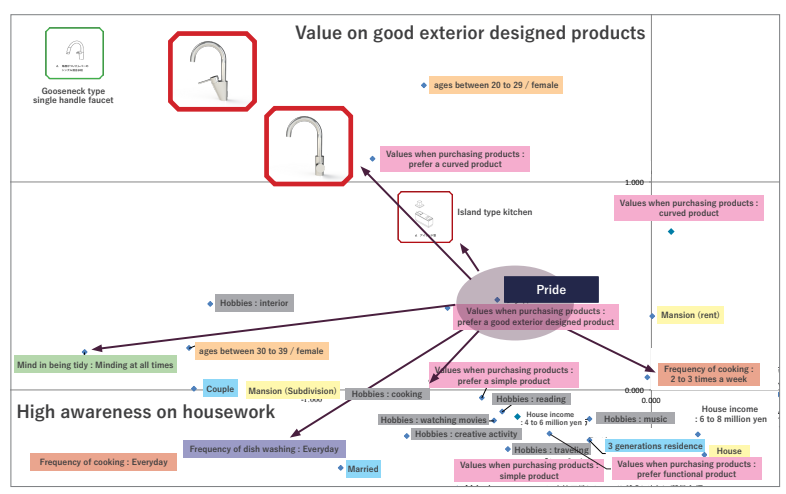

Figure 1: Enlarged scatter plot focused on "Pride"

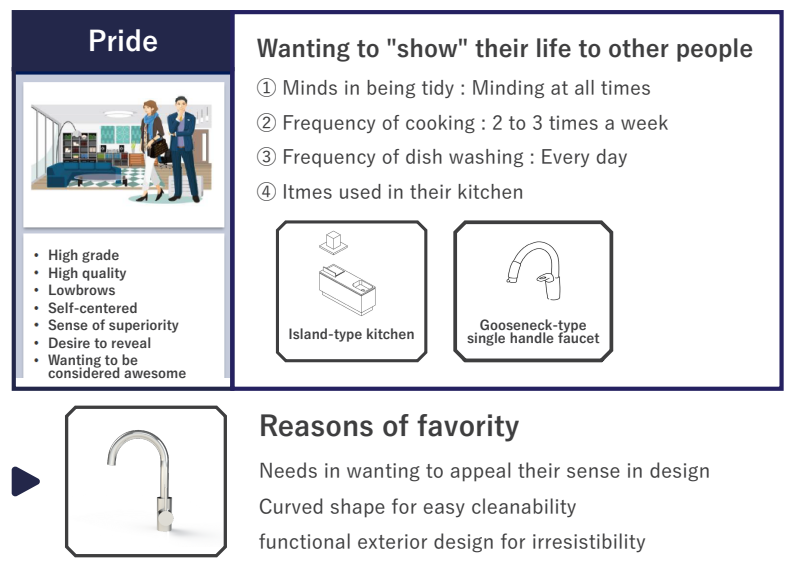

Figure 2: Summary on the "Pride" attribute

\section{Research on abstracting the images of single handle faucets which improves the purchase intention of the specific lifestyle segment}

\subsection{Survey content and purpose}

In the previous research, we were able to abstract the attributes who values most on the exterior designs, when purchasing a single handle faucet. As the next step, we were required to find out what kind of images are correlated to their purchase intentions, in order to make a design guideline. Therefor, a questionnaire survey was developed, in the purpose of abstracting the images of single handle faucets, which improves the purchase intention of our target attribute. We made the target attributes, evaluate 5 pair of image words and their purchase intention, using 7 basic form of single handle faucets.

\subsection{Survey method}

7 basic form of single handle faucets, which are different in spout shape and handle position were developed. The size and configurations were decided from the existing single handle faucets on the market. We abstracted 22 test subjects who fulfill the requirements of our target attribute, and made them evaluate those faucets, with 5 pair of image words in 6 levels, using the semantic differential method. This survey was simultaneously implemented with the research from the chapter before. After that, we used the multiple regression analysis, to abstract the images which improves their purchase intention.

\subsection{Result}

Table 1 shows the result of the multiple regression analysis. As the numerical value of the correlation coefficient shown in the table, the image words which improves target attribute is, "simple", "high grade" and "high design". From the test on partial regression coefficient, the result on "simple" and "high grade" shown its significance. However, the result on the "highly functional - high design" has not shown its significance. We speculated this result came from the target attribute's needs on the design were nearly equal to the meaning as "a functional exterior design for irresistibility", from the previous research. Therefor, these image words have not satisfied the requirements as a pair for our target attribute. From these results, we were able to abstract the image words which improves the target attribute's purchase intention, are "simple" and "high grade". 
Table 1: Analysis result on the correlation between purchase intention and other images

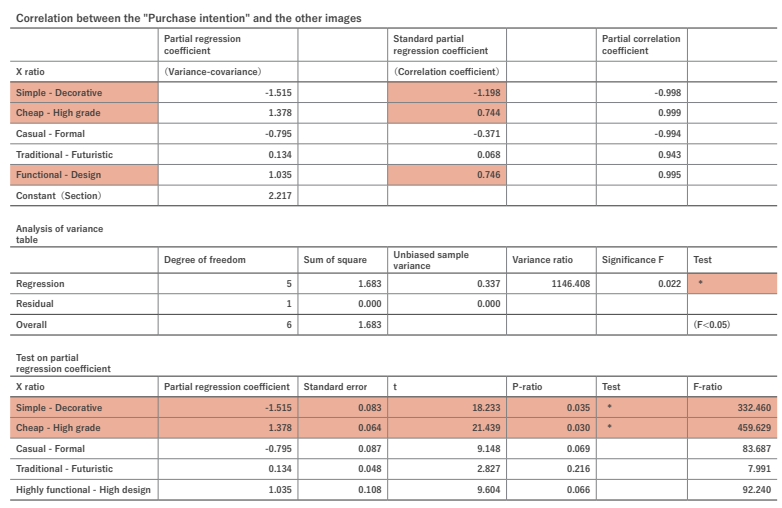

4. Research on abstracting the design elements of single handle faucets which improves the purchase intention and the correlated images

\subsection{Survey content and purpose}

In the previous research, we were able to abstract the image words which has a strong correlation, with our target attribute's purchase intention. As the next step, we were required to abstract the specific design elements, which has a strong correlation with the abstracted image words and purchase intention, to complete our design guideline. Therefor, a questionnaire survey was developed, in the purpose of abstracting the specific design elements, which has a strong correlation with those image words and the purchase intention of our target attribute. We made the target attributes, evaluate the abstracted image words from the previous research and their purchase intention, using 21 existing single handle faucets with various design elements on the market.

\subsection{Survey method}

We abstracted 7 test subjects who fulfill the requirements of our target attribute, and made them evaluate 21 existing gooseneck single handle faucets, with 2 pair of image words and their purchase intention in 6 levels, using the semantic differential method. After that, we used the mathematical quantification theory class I, to abstract the specific design elements, which has a strong correlation with the abstracted image words and purchase intention of our target attribute.

\subsection{Result}

Table 2 to Table 4 shows each result of the mathematical quantification theory class I. As the standard category score shown in each table, there are no exceptionally high score. Therefor, all of the analysis results are safely said to have significance. Furthermore, as the ratio of the multiple correlation coefficient and coefficient of determination shown in each table, each analysis result is safely said to have high prediction accuracy. According to this analysis, we found out the design elements, which has strong correlations with the target attribute's purchase intention, "simple" and "high grade" images are, "Curve direction of the Spout tip : Downward direction", "Smoothness of the Joints between the Handle part and Mounting part : NOT Smooth", "Smoothness of the Joints between the Mounting part and Spout : Smooth", "Ratio of the Faucet (from Mounting part to Spout tip) : Mounting part (Thick) $\rightarrow$ Center (Thin) $\rightarrow$ Spout tip (Thick)", "Cartridge shell : NOT Dividing the Mounting part and spout", "Shape of spout (from the cross-section) : Circle" and "Overall impression : Geometric". From these results, we were able to abstract the design elements which improves the target attribute's purchase intention, are "simple" and "high grade" images. Table 5 shows the abstracted design elements to improve our target attribute's purchase intention, "simple" and "high grade" image.

Table 2: Analysis result on the correlation between purchase intention and design elements

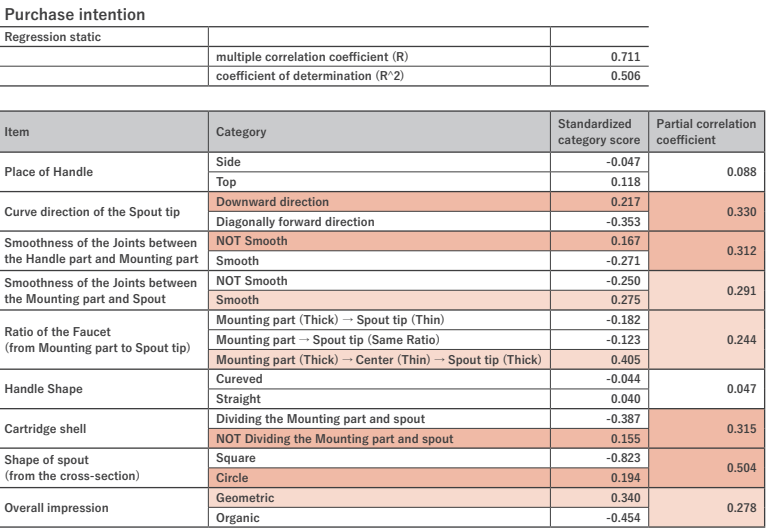

Table 3: Analysis result on the correlation between simple image and design elements

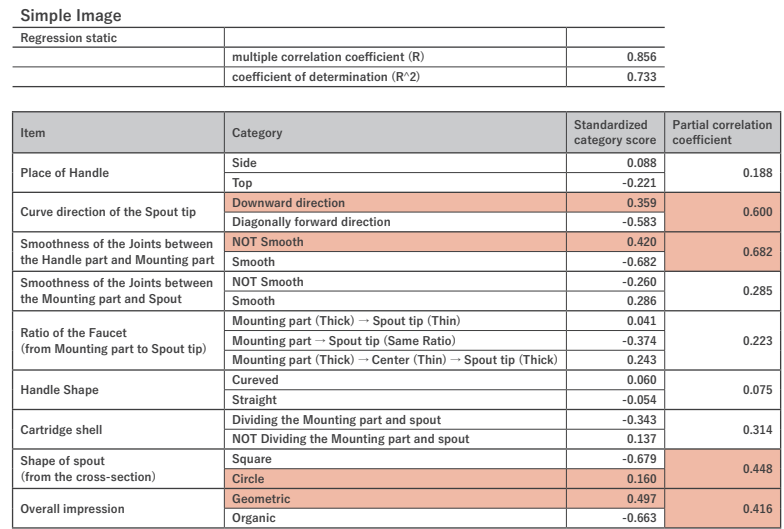


Table 4: Analysis result on the correlation between high grade image and design elements

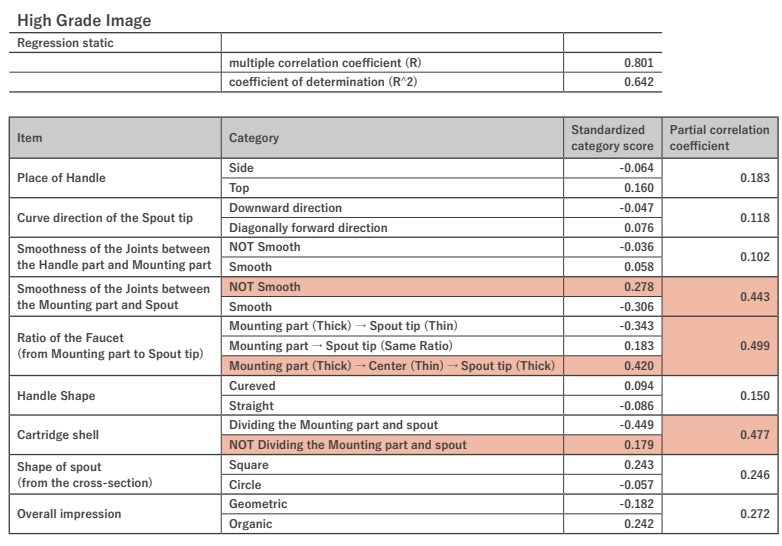

Table 5: The abstracted design elements for improving the target attribute's purchase intention

\begin{tabular}{|l|l|}
\hline Curve direction of the Spout tip & Downward direction \\
\hline $\begin{array}{l}\text { Smoothness of the Joints between the } \\
\text { Handle part and Mounting part }\end{array}$ & NOT Smooth \\
\hline $\begin{array}{l}\text { Smoothness of the Joints between the } \\
\text { Mounting part and Spout }\end{array}$ & Smooth \\
\hline $\begin{array}{l}\text { Ratio of the Faucet } \\
\text { (from Mounting part to Spout tip) }\end{array}$ & $\begin{array}{l}\text { Mounting part (Thick) } \rightarrow \text { Center (Thin) } \\
\rightarrow \text { Spout tip (Thick) }\end{array}$ \\
\hline Cartridge shell & NOT Dividing the Mounting part and spout \\
\hline $\begin{array}{l}\text { Shape of spout } \\
\text { (from the cross-section) }\end{array}$ & Circle \\
\hline Overall impression & Geometric \\
\hline
\end{tabular}

\section{Conclusion}

In this study, we abstracted the design elements of single handle faucets, which improves the purchase intention and the correlated images, of the specific lifestyle segment which values most on the designs when purchasing. First, we developed 7 basic designed single handle faucets and had a questionnaire survey on the test subject's basic attributes, lifestyle and image evaluation on the developed faucets. We used the mathematical quantification theory class III, to find out what kind of basic attributes and lifestyles values most on the designs of the single handle faucet when purchasing, and what kind of basic design configuration they prefer most. From this analysis, we found out the lifestyle segment, which values most on the designs of the single handle faucet when purchasing, and has a high awareness on doing housework, is the lifestyle segment named "Pride", regarding their personality as "wanting to show their life to other people". Furthermore, the design configuration that the "Pride with high awareness on housework" prefer most, is the type which is called the gooseneck faucet. Secondly, we used the multiple regression analysis, to abstract the image words, which has a strong correlation with the purchase intention, regarding to the abstracted basic attributes and the lifestyle above. According to this analysis, we found out the image words which has a strong mutual relation with the appearance are "simple" and "high grade". Therefore, these image words will help to develop designs of the gooseneck-type single handle faucets and improve the purchase intention. Finally, we made a questionnaire survey on the images of the existing gooseneck-type single handle faucet with various design elements. We gathered the test subjects with the lifestyle which was discriminated as "Pride", and has a strong awareness to housework, and made them evaluate on 21 existing gooseneck-type single handle faucet's images on "simple", "high grade" and their "purchase intention". We used the mathematical quantification theory class I, to abstract the design elements, which has a strong correlation with "simple" and "high grade" image, and their "purchase intention". According to this analysis, we found out the design elements, which has strong correlations with the items above are, "Curve direction of the Spout tip : Downward direction", "Smoothness of the Joints between the Handle part and Mounting part : NOT Smooth", "Smoothness of the Joints between the Mounting part and Spout : Smooth", "Ratio of the Faucet (from Mounting part to Spout tip) : Mounting part (Thick) $\rightarrow$ Center (Thin) $\rightarrow$ Spout tip (Thick)", "Cartridge shell : NOT Dividing the Mounting part and spout", "Shape of spout (from the cross-section) : Circle" and "Overall impression : Geometric". In conclusion, we were able to make a design guideline for designing single handle faucets based on the correlations between human lifestyle and design elements. We believe our study will make the design work easier and adjustable for designing single handle faucets, targeting people who makes the trends, and values most on the appearance. Moreover, help spread the designed product efficiently to the world. Furthermore, we believe the analytical steps we took in purpose of marketing and designing, can contribute in developing design guideline for various consumer products in various fields, and spread newly developed products to the world efficiently than the conventional design process.

\section{ACKNOWLEDGMENT}

This work was supported by TAKAGI Co. Ltd., of Japan.

\section{REFFERENCES}

[1] FORWARD CO.LTD.,; Consumer Segmentation of Values Flower Profile Book 2018, 2018.

[2] Riku Takagi, Keita Sasaya, Ippei Shimizu and Noriko Hashida; Study on the single handle faucet's body configuration difference which affects human emotions, Kansei-Kougaku, 2019. 